\title{
Influence of modulation of pump and seed signals on fiber amplification of broadband pulses
}

\author{
K. Gürel, P. Elahi, I. L. Budunoğlu, Ç. Şenel, P. Paltani and F. Ö. Ilday \\ Department of Physics, Bilkent University, TR-06800 Ankara, Turkey \\ k_gurel@ug.bilkent.edu.tr
}

\begin{abstract}
We report on characterization of the transfer of pump and seed signal modulations, including noise, during fiber amplification. We demonstrate experimentally and theoretically that pump (signal) modulations are transferred only below (above) a cut-off frequency.
\end{abstract}

(C) 2010 Optical Society of America

OCIS codes: (060.7140) Ultrafast processes in fibers; (140.3538) Lasers pulsed; (320.7090) Ultrafast Lasers.

Rapid progress is being reported on the development of high-power fiber laser-amplifier systems. However, despite its importance in applications, intensity noise of fiber amplifiers has not received a close scrutiny. Recently, we reported a systematic study of intensity noise of mode-locked fiber oscillators [1]. A key conclusion of this study was that, generally, there is a trade-off between the noise level of the oscillator and the highest pulse energy that can be extracted. Thus, the route to low-noise high-power operation could be to operate the oscillator at moderate power levels and use external amplification. A major contributor to noise during amplification is the transfer of noise of the pump lasers, which is particularly severe with high-power multimode pump lasers. In addition to noise considerations, there are various applications where modulations of the signal or the pump need to be transferred to the amplified signal or an existing modulation to be preserved, such as broadband carrier-envelope phase (CEP) stabilization. Thus, it is important to understand the dynamics of signal and pump modulations during fiber amplification. Here, we present a simple, yet accurate theoretical model for transfer of signal or pump modulations using a modulation transfer function approach, the results of which are verified experimentally. We then apply the knowledge of these dynamics to predict the noise of an amplified pulse train.

We model modulation of the pump and signal powers as well as population levels at frequency $\omega=2 \pi v$ as:

$$
\begin{gathered}
P_{p(s)}(z, t)=P_{p(s), 0}(z)\left(1+q_{p(s)}(z, \omega) e^{i \omega t}\right)+c . c . \\
n_{1(2)}(z, t)=n_{1(2), 0}(z)\left(1+q_{1(2)}(z, \omega) e^{i \omega t}\right)+c . c .,
\end{gathered}
$$

where $P_{p(s)}(z, t)$ are the pump (signal) powers and $n_{1(2)}(z, t)$ are the fractional population densities of the lower (upper) states, and $q(\omega, z)$ represent the modulation amplitudes. $P_{p(s), 0}(z)$ and $n_{1(2), 0}(z)$ correspond to solutions without modulation. Eqn.'s (1) and (2) along with the rate equations are solved numerically following the well-known model in [2]. The modulation transfer functions (MTF's), $\xi_{p}(v)$ for pump and $\xi_{s}(v)$ for signal are given by:

$$
\xi_{p(s)}(v)=\frac{q_{s(s)}(L, \omega)}{q_{p(s)}(0, \omega)}
$$

For experimental verification of the model, we use a similariton Yb-doped fiber laser (repetition rate of $35 \mathrm{MHz}$ ). The seed signal is split by a fiber coupler, where one port is directed to a high-resolution power meter and the other port seeds a forward-pumped fiber amplifier comprising of $120 \mathrm{~cm}$-long Yb-doped fiber with an absorption peak of $200 \mathrm{~dB} / \mathrm{m}$ at $976 \mathrm{~nm}$. The amplified signal is separated from any unabsorbed pump light using a wavelength division multiplexer. The MTF's are measured by modulating the pump and seed power by directly modulating the pump diode's current and through an acousto-optic modulator, respectively, over $1 \mathrm{~Hz}-250 \mathrm{kHz}$. The MTF is obtained via the same method as in [3]. However, the entire measurement is automated via a computer.

Measured and calculated MTF's for the case of $1 \mathrm{~mW}$ of seed and $100 \mathrm{~mW}$ of pump power are illustrated in Fig. 1(a). The MTF's are fit very well by generalized Lorentzian functions, given by $\xi_{p}(v)=\xi_{p, 0} /\left(1+\left(v / v_{p, 0}\right)^{n}\right)$ for the pump and $\xi_{s}(v)=1-\left(1-\xi_{s, 0}\right) /\left(1+\left(v / v_{s, 0}\right)^{n}\right)$ for the seed. Here, $\xi_{p, 0}$ and $\xi_{s, 0}$ represent the small frequency transfer coefficients and, $v_{p}$ and $v_{s}$ are the $3-\mathrm{dB}$ cutoff frequencies for pump and signal, respectively. A prerequisite for using the transfer function approach is that the system under study must be linear. Indeed, the small-frequency 


\section{CThU5.pdf}
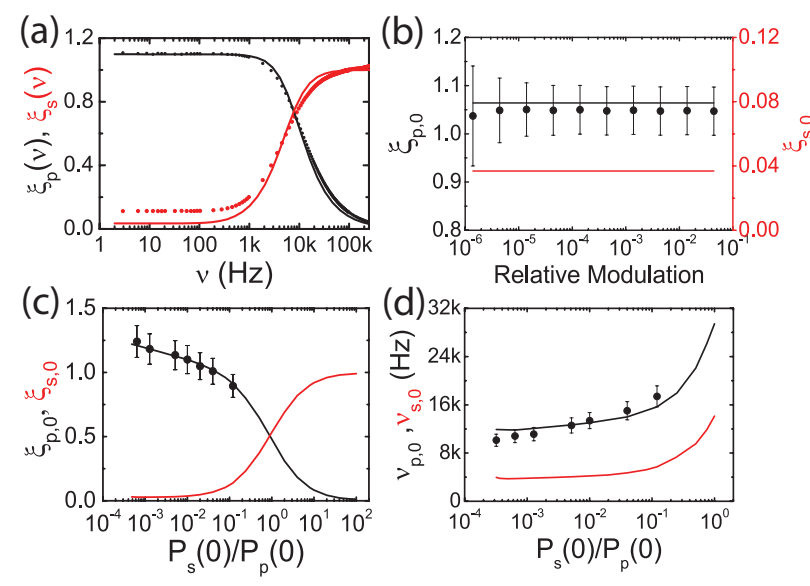

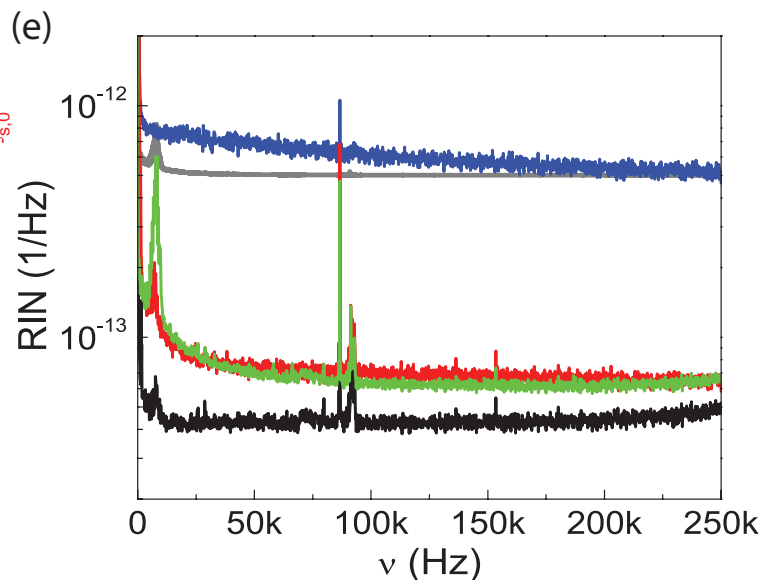

Fig. 1. (a) Measured (dotted) and calculated (solid line) MTF curves for pump (black) and seed (red). (b) $\xi_{p, 0}$ and $\xi_{s, 0}$ versus the modulation depth. (c) $\xi_{p, 0}$ and $\xi_{s, 0}$ versus seed-pump power ratio. (d) $v_{p, 0}$ and $v_{s, 0}$ versus seed-pump power ratio. (e) Measured RIN spectra of the seed (black), ASE (blue), amplifier output (red) and calculated RIN spectra of ASE (gray) and amplifier output (green).

transfer coefficients, $\xi_{p, 0}$ and $\xi_{s, 0}$, are verified to be independent of the modulation depth over a large range of seed power to pump power ratios (Fig. 1(b)). The dependence of the small-frequency transfer coefficients, and the 3-dB cutoff frequencies, $v_{p, 0}$ and $v_{s, 0}$ on the ratio of the launched seed power to the pump power are shown in Fig. 1(c) and (d). Importantly, the transfer coefficients for pump and seed always approach 0 and 1 , respectively, at high frequencies, meaning that high-frequency pump noise has practically no influence on amplifier performance. Conversely, any seed modulations are strongly suppressed at low frequencies due to the saturation effect. As the signal and pump MTF's are complementary, rendering possible broadband modulation (e.g., for CEP stabilization) by modulating both the seed and the pump at high and low frequencies, respectively.

Next, we apply the knowledge of the MTF's to predict relative intensity noise (RIN) of a fiber amplifier, which is taken to be due to the noise of the seed, the pump and excess noise due to quantum fluctuations of amplified spontaneous emission (ASE) in the amplifier. To first order, ASE RIN is simply inversely proportional to the optical bandwidth of the ASE spectrum [4]. The pump and seed RIN contributions, weighted by their respective MTFs, and RIN due to ASE are all added to arrive at the amplifier RIN. This approach ignores any interaction between these noise sources, which is justified as long as the noise levels are low. As an example, Fig. 1(e) shows the measured RIN of the seed, pump, amplifier output (seed and pump powers of $12 \mathrm{~mW}$ and $100 \mathrm{~mW}$, respectively) as well as ASE (no seed). Calculated RIN for strong seed and ASE reveal very good agreement with the experimental results. The integrated (from $1 \mathrm{kHz}$ to $250 \mathrm{kHz}$ ) RIN of the seed source, the measured amplifier RIN and the calculated amplifier RIN are $0.015 \%, 0.019 \%$ and $0.019 \%$, respectively, showing an excellent agreement of the measurements and calculations.

In summary, we have characterized, for the first time to our knowledge, the transfer functions for seed and pump modulations to the output of a Yb-fiber amplifier. We developed a simple theoretical model describing these dynamics, which shows excellent agreement with the experiments. We find that seed (pump) modulations are not transferred at low (high) frequencies. If broadband modulation is desired, it is necessary to modulate both the seed and the pump at high and low frequencies, respectively. We use our model to predict the intensity noise of an amplifier, obtaining excellent agreement with experiments. Finally, we show that fiber amplification can be achieved with little excess noise if the low-frequency noise of the pump is suppressed and the seed is strong enough to suppress ASE. Armed with our model, design of high-power, low-noise fiber laser systems can be guided by theory with high accuracy.

\section{References}

1. I. L. Budunoglu, C. Ülgüdür, B. Oktem, and F. Ö. Ilday, "Intensity noise of mode-locked fiber lasers," Opt. Lett. 34, 2516 (2009).

2. C. R. Giles and E. Desurvire, "Modeling erbium-doped fiber amplifiers," J. Lightwave Technol. 9 (2), 271 (1991).

3. T. D. Mulder, R. P. Scott, B. H. Kolner, "Amplitude and envelope phase noise of a modelocked laser predicted from its noise transfer function and the pump noise power spectrum," Opt. Express, 16, 14186 (2008).

4. H. Hodara, "Statistics of thermal and laser radiation," Proc. IEEE 53, 696 (1965). 